\title{
On curl-preserving finite volume discretizations for shallow water equations
}

\section{Conference Paper}

\section{Author(s):}

Jeltsch, Rolf; Torrilhon, Manuel

Publication date:

2006

Permanent link:

https://doi.org/10.3929/ethz-b-000035518

Rights / license:

In Copyright - Non-Commercial Use Permitted

Originally published in:

BIT 46(Supplement 1), https://doi.org/10.1007/s10543-006-0089-5 


\title{
ON CURL-PRESERVING FINITE VOLUME DISCRETIZATIONS FOR SHALLOW WATER EQUATIONS*
}

\author{
ROLF JELTSCH ${ }^{1}$ and MANUEL TORRILHON ${ }^{2}$ \\ ${ }^{1}$ Seminar for Applied Mathematics, Swiss Federal Institute of Technology (ETH) Zurich, \\ 8092 Zurich, Switzerland. email: jeltsch@math.ethz.ch \\ ${ }^{2}$ Applied and Computational Mathematics, Princeton University, Princeton, NJ, USA. \\ email: mtorrilh@princeton.edu
}

Dedicated to Björn Engquist on the occasion of his 60th birthday.

\begin{abstract}
.
The preservation of intrinsic or inherent constraints, like divergence-conditions, has gained increasing interest in numerical simulations of various physical evolution equations. In Torrilhon and Fey, SIAM J. Numer. Anal. (42/4) 2004, a general framework is presented how to incorporate the preservation of a discrete constraint into upwind finite volume methods. This paper applies this framework to the wave equation system and the system of shallow water equations. For the wave equation a curl-preservation for the momentum variable is present and easily identified. The preservation in case of the shallow water system is more involved due to the presence of convection. It leads to the vorticity evolution as generalized curl-constraint. The mechanisms of vorticity generation are discussed.

For the numerical discretization special curl-preserving flux distributions are discussed and their incorporation into a finite volume scheme described. This leads to numerical discretizations which are exactly curl-preserving for a specific class of discrete curl-operators.

The numerical experiments for the wave equation show a significant improvement of the new method against classical schemes. The extension of the curl-free numerical discretization to the shallow water case is possible after isolating the pressure flux. Simulation examples demonstrate the influence of the modification. The vortex structure is more clearly resolved.
\end{abstract}

AMS subject classification (2000): 65M06, 74S10.

Key words: finite volume methods, vorticity, shallow water equations, constraint preservation.

\section{Introduction.}

The partial differential equations of electromagnetism and shallow water flows describe fundamentally different physical processes. Mathematically, however,

\footnotetext{
^ Received October 30, 2005. Accepted March 23, 2006. Communicated by Anders Szepessy.
} 
both systems of equation show an interesting structural similarity: an intrinsic constraint equation which follows from a vector-differential identity of the evolution operator. In electromagnetism the divergence of the magnetic field is preserved, while shallow water flows provide a preservation of the curl of velocity, that is, vorticity.

Indeed, many evolution equations in physics and engineering come along as partial differential equations with intrinsic constraints. These constraints are characterize by local differential relations for the variables which follow solely from the evolution equation and do not form an independent equation. Thus, they are inherent or intrinsic to the evolution operator. Dafermos refers to those constraints as involutions in [3]. While the constraints are exactly satisfied at the analytical level, constraint violation is a major issue in numerical calculations. Both from a theoretical and practical point of view it is preferable to design numerical method that obey intrinsic constraint on a discrete level. However, such constraints challenge numerical methods since discrete preservation properties are not easily established. Special methods are needed.

The introduction mixed finite elements in [16] provides the possibility to handle solenoidal or curl-free fields in finite elements simulations and is widely used. Also, so-called mimetic finite difference discretizations have been developed in [8] which are used mainly for electromagnetism. However, most fluid dynamics simulations require finite volume methods, which deal with piece-wise constant data and upwind directions. This makes it necessary to use additional approaches to assure constraint preservation. Most severe problems occur in simulations of magnetohydrodynamics where the magnetic field has to be kept divergence-free, see [1]. Hence, many specialized finite volume methods have been proposed, see e.g., [4] or [19].

A general numerical constraint preserving framework for finite volume schemes has been developed in [18] and applied to magnetohydrodynamics, see [17]. This paper transfers the results and methods to the case of vorticity preservation in shallow water flows. Section 3 of this paper gives a brief introduction into the flux distribution concept of [18]. In contrast to the divergence-free condition of the magnetic field, the vorticity preservation is a non-linear constraint since it follows only from the Lagrangian form of the equations. It is also not a pure preservation but super-imposed by advection. Details are given in Section 2 . The shallow water equations are related to the non-linear wave equation system and, hence, Section 4 presents numerical results for curl-preserving methods for the wave equation system. In Section 5 these numerical methods are extended to the shallow water case.

\section{Constrained partial differential equations.}

We will briefly introduce the generic form of intrinsic constraints and discuss physical examples of fluid-type equations with focus on the shallow water equations. 


\subsection{Generic cases.}

In the generic case we consider a time dependent vector field $\mathbf{u}: \mathbb{R}^{D} \times \mathbb{R}^{+} \rightarrow$ $\mathbb{R}^{D}$ in $D$-space. Two intrinsically constrained evolution equations together with the constraints itself are then given by

(B) $\partial_{t} \mathbf{u}+\operatorname{grad} F_{B}(\mathbf{u})=0$

$\operatorname{div} \mathbf{u}=$ const

curl $\mathbf{u}=$ const,

where $\mathbf{F}_{A}$ and $F_{B}$ are appropriate flux functions. The evolution operators are formed such that they exactly vanish under the application of the constraints due to vector-differential identities. Hence, any initial distribution of $\operatorname{div} \mathbf{u}$ or curl $\mathbf{u}$ respectively, is perfectly preserved during the entire evolution. Constrained evolutions of both types, $(A)$ and $(B)$, can be found in physics.

\subsection{Wave equation system.}

The first numerical part of this paper will consider the wave equation as a first order system which is found to have a constrained evolution of type $(B)$. The variables are then given by $U=\{\rho, \mathbf{m}\} \in \mathbb{R}^{1+D}$, with a density $\rho$ and a momentum variable $\mathbf{m}$. The wave equation system reads

$$
\begin{array}{r}
\partial_{t} \rho+\operatorname{div} \mathbf{m}=0 \\
\partial_{t} \mathbf{m}+\operatorname{div}(p(\rho) \mathbf{I})=0,
\end{array}
$$

where the pressure function $p(\rho)$ with $p^{\prime}(\rho)>0$ must be given as a function of the density in order to close the system. The linear wave equation is recovered by choosing $p(\rho)=\rho$ and eliminating $\mathbf{m}$ in $(2.2)$.

Since $\operatorname{div}(p(\rho) \mathbf{I})=\operatorname{grad} p(\rho)$ the wave equation system has the constraint $\operatorname{curl} \mathbf{m}=$ const for the vorticity $\operatorname{curl} \mathbf{m}$. The system (2.2) can be viewed as model for the Euler equations or shallow water equations if the inertial terms of the momentum are neglected in these models. Indeed, both systems exhibit a kind of vorticity constraint superimposed by a pure advection, see e.g., [14].

\subsection{Shallow water equations.}

The wave equation system (2.2) can be considered as a simple model for inviscid fluid flow when convection is neglected. The remaining phenomenon are sound waves. Accordingly, the constraint properties of the wave equation system can be found in the equations of fluid dynamics as well. However, the presence of convection complicates the issue considerably. The second numerical part of the paper will investigate the shallow water equations which form a special case of inviscid fluid dynamics.

In the following the fluid variables of interest are the density $\rho$ and velocity $\mathbf{v}$ in one, two or three space dimensions. The field equations are given by the conservation laws of mass and momentum. They read

$$
\begin{array}{r}
\partial_{t} \rho+\operatorname{div} \rho \mathbf{v}=0 \\
\partial_{t}(\rho \mathbf{v})+\operatorname{div}\left(\rho \mathbf{v} \cdot \mathbf{v}^{T}+p \mathbf{I}\right)=0
\end{array}
$$


with a general pressure law

$$
p=p(\rho, T)
$$

The temperature $T$ follows from an energy equation. The case $T=$ const, hence, $p=p(\rho)$ produces a closed system (2.3) which represents various basic fluid models. For example, $p(\rho)=c^{2} \rho$ generates the isothermal Euler equations where the constant $c$ is the sound speed. The case of shallow water equations is introduced by choosing $p(\rho)=\frac{1}{2} g \rho^{2}$ where $g$ is the gravitational constant.

As in the case of the wave equation system the momentum variable $\mathbf{m}=\rho \mathbf{v}$ and its curl can be considered. However, due to the convection in $(2.3 \mathrm{~b})$ no proper preservation property of curl $\mathbf{m}$ can be found. Instead of the momentum it is more interesting to consider the vorticity

$$
\boldsymbol{\Omega}=\operatorname{curl} \mathbf{v}
$$

of the velocity $\mathbf{v}$ alone. Note, that for the wave equation system also the vorticity for the velocity $\mathbf{m} / \rho$ is preserved.

For smooth solutions it is easy to derive well known evolution equations which replace the curl-preservation of the wave equation system. If the momentum balance $(2.3 \mathrm{~b})$ is written in Lagrangian form with help of the mass balance and the convection expression is substituted by $\mathbf{v} \cdot \operatorname{grad} \mathbf{v}=\operatorname{grad} \frac{1}{2} \mathbf{v}^{2}-\mathbf{v} \times \operatorname{curl} \mathbf{v}$ we obtain

$$
\partial_{t} \mathbf{v}+\operatorname{grad} \frac{1}{2} \mathbf{v}^{2}+\frac{1}{\rho} \operatorname{grad} p=\mathbf{v} \times \boldsymbol{\Omega}
$$

for the velocity. Taking the curl leads to

$$
\partial_{t} \boldsymbol{\Omega}+\operatorname{curl}(\boldsymbol{\Omega} \times \mathbf{v})=\frac{1}{\rho^{2}} \operatorname{grad} \rho \times \operatorname{grad} p,
$$

an evolution equation for vorticity. In the context of constraints, Equation (2.7) represents a generalized constraint. The equation follows from the system (2.3) alone and is not an independent equation. Hence, it is reasonable to ask if it is satisfied in numerical simulations. However, it is an evolution equation and not a local equation as in the generic setting (2.1). This makes it difficult to verify numerically.

In the case $p=p(\rho)$ the right hand side of (2.7) vanishes and it follows an advection equation for vorticity. The curl-type advection operator is discussed in [18]. In such a setting the vorticity is preserved but advected. Furthermore, if it is zero initially, $\left.\boldsymbol{\Omega}\right|_{t=0} \equiv 0$, it vanishes for all times in an inviscid solution as long as the solution remains smooth. Viscous terms and boundaries lead to additional vorticity sources proportional to $\operatorname{grad} \rho \times \Delta \mathbf{v}$ which will not be discussed here.

For the shallow water case $(2.6)$ can be written nicely as

$$
\partial_{t} \mathbf{v}+\operatorname{grad}\left(\frac{1}{2} \mathbf{v}^{2}+g \rho\right)=\mathbf{v} \times \boldsymbol{\Omega} .
$$


From here the advective preservation of the vorticity is obvious. Hence, in numerical meteorology, where vorticity preservation is crucial, the form (2.8) is commonly used in discretizations, see [14]. However, (2.8) is not in conservation form and numerical models using this equation will fail to produce correct shallow water shock waves and shock speeds. In meteorological calculations shock waves do not occur.

In two dimensions we consider $\mathbf{v}=\left(v^{(x)}, v^{(y)}, 0\right)^{T}$ and the vorticity has only a single non-vanishing entry

$$
\omega=(\boldsymbol{\Omega})_{z}=\partial_{x} v^{(y)}-\partial_{y} v^{(x)} .
$$

From (2.7) for the shallow water equations we obtain

$$
\partial_{t} \omega+\operatorname{div}(\omega \mathbf{v})=0
$$

for the scalar vorticity. This is the constraint equation which corresponds to curl $\mathbf{m}=$ const in the wave equation case. We note, that for smooth solutions vorticity can not be generated. Existing vorticity distributions are not frozen but only advected.

In the presence of discontinuous solutions things change for the vorticity. In fact, shocks can generate vorticity when interacting with a density gradient. This is true for any fluid model. Here, we consider only the shallow water equation but the expressions derived for the vorticity generation across a shock are more general.

The Rankine-Hugoniot jump conditions are given by

$$
\begin{aligned}
{\left[\rho v^{(x)}\right] } & =0 \\
{\left[\rho v^{(x)} v^{(x)}+\frac{1}{2} g \rho^{2}\right] } & =0 \\
{\left[v^{(y)}\right] } & =0
\end{aligned}
$$

for a shock wave with $\rho v^{(x)} \neq 0$. From them we derive the relations for the quantities before and after the shock, viz.

$$
\rho_{1} / \rho_{0}=\frac{1}{2}\left(\sqrt{1+8 M^{2}}-1\right), \quad v_{1}^{(x)} / v_{0}^{(x)}=2\left(\sqrt{1+8 M^{2}}-1\right)^{-1}
$$

with the Froude or Mach number $M=v_{0}^{(x)} / \sqrt{g \rho_{0}}$. The jump of the vorticity is denoted by

$$
\Delta \omega=\left[\partial_{x} v^{(y)}-\partial_{y} v^{(x)}\right] .
$$

In special situations it may occur that the vorticity is vanishing on one side of the shock but non-zero on the other. This is the most obvious case of vorticity generation.

In order to derive an expression for $\Delta \omega$ we consider a shock wave propagating in $x$-direction in a non-homogeneous two-dimensional environment. The calculation follows the work [12] which relies on [20]. After transforming the observation 
frame to a stationary infinitesimal vicinity of a point on the shock wave we consider the momentum equation in Lagrangian form in the smooth regions left and right to the shock wave. For the $y$-component it follows

$$
\rho \mathbf{v} \cdot \operatorname{grad} \mathbf{v}+\operatorname{grad} p=0 \Rightarrow \rho v^{(x)} \partial_{x} v^{(y)}+\rho v^{(y)} \partial_{y} v^{(y)}+\partial_{y} p=0 .
$$

Taking the jump over the discontinuity and using the Rankine-Hugoniot conditions we derive

$$
\rho v^{(x)}\left[\partial_{x} v^{(y)}\right]+[\rho] v^{(y)} \partial_{y} v^{(y)}+\left[\partial_{y} p\right]=0,
$$

which is an expression for the first part of $\Delta \omega$, i.e., $\left[\partial_{x} v^{(y)}\right]$. The second part follows from differentiating the Rankine-Hugoniot condition itself yielding

$$
0=\partial_{y}\left[\rho v^{(x)} v^{(x)}+p\right]=\partial_{y}\left(\rho v^{(x)}\right)\left[v^{(x)}\right]+\rho v^{(x)}\left[\partial_{y} v^{(x)}\right]+\left[\partial_{y} p\right]
$$

where $\left[\rho v^{(x)}\right]=0$ was used. Subtracting both equations leads to

$$
\Delta \omega=\frac{\partial_{y}\left(\rho v^{(x)}\right)}{\rho v^{(x)}}\left[v^{(x)}\right]-\frac{v^{(y)} \partial_{y} v^{(y)}}{\rho v^{(x)}}[\rho]
$$

which is an expression for the jump of vorticity through a shock in dependence of tangential gradients and jumps of density and velocity. As long as the field in front of the shock are homogeneous no vorticity will by generated. Assuming a non-varying velocity in front of the shock we find the compact relation

$$
\Delta \omega=\frac{\partial_{y} \rho_{0}}{\rho_{0}}\left[v^{(x)}\right] .
$$

This is the well-known result that a shock wave which hits a tangential density gradient component will generate vorticity.

\section{Constraint-preserving finite-volume methods.}

The idea of constraint preserving numerical methods originated to a considerable extent in the context of magnetohydrodynamics where the divergence constraint produces severe numerical problems, see [1, 4] and the review [19].

Recently, a general framework for constraint preserving finite volume methods have been presented in [18], see also [5]. It has been successfully applied to the MHD system in [17]. In the following we will discuss the application of the framework to curl-preservation in order to use it for the wave equation system (2.2) and shallow water system (2.3). Vorticity-preserving finite difference methods for the wave equation system are also discussed in [15].

\subsection{Flux distributions.}

Following the presentation in [18] we consider a generic vector field $\mathbf{u} \in \mathbb{R}^{D} \rightarrow$ $\mathbb{R}^{D}$. It is approximated on a finite volume grid $\mathcal{T}=\left\{K_{i}\right\}_{i \in \mathbb{N}}$ consisting of non- 
overlapping cells $K_{i}$ by a cell-wise constant vector-valued grid function $\tilde{\mathbf{u}} \in$ $V=\left\{g: \mathcal{T} \rightarrow \mathbb{R}^{D}\right\}$. For the numerical evolution of the vector field a certain finite volume update should be given in which generally each cell contributes to neighboring cells by fluxes during one time step. In order to identify the constraint preserving properties of such an update we need to introduce a special formulation based on so-called flux distributions.

Definition 3.1 (Flux distribution). We define a "flux distribution" $\boldsymbol{\Phi}_{K}$ : $V \rightarrow V$ which is attached to a grid cell $K$. It maps the grid function $\tilde{\mathbf{u}}$ into another grid function, that is $\boldsymbol{\Phi}_{K}(\tilde{\mathbf{u}}) \in V$ with $\operatorname{supp}\left(\boldsymbol{\Phi}_{K}(\tilde{\mathbf{u}})\right)=K \cup \bigcup_{\widehat{K} \in \mathcal{N}(K)} \widehat{K}$. The evaluation $\left.\boldsymbol{\Phi}_{K}(\tilde{\mathbf{u}})\right|_{\widehat{K}}$ gives the change of $\tilde{\mathbf{u}}$ at cell $\widehat{K}$ caused by cell $K$ during a time step, that is the flux.

Any finite volume scheme can be written in the form

$$
\left.\tilde{\mathbf{u}}^{m+1}\right|_{K}=\left.\tilde{\mathbf{u}}^{m}\right|_{K}+\left.\sum_{\widehat{K} \in\{K\} \cup \mathcal{N}(K)} \boldsymbol{\Phi}_{\widehat{K}}\left(\tilde{\mathbf{u}}^{m}\right)\right|_{K}
$$

once the specific flux distributions have been identified. Here $\mathcal{N}(K)$ defines the neighboring cells of $K$. The flux distribution represents a special shape of the change of $\tilde{\mathbf{u}}$ associated with a generic cell during one time step. The formulation (3.1) requires the decomposition of the total change of $\tilde{\mathbf{u}}$ into a superposition of flux distributions associated to neighboring cells.

The object $\boldsymbol{\Phi}_{K}(\tilde{\mathbf{u}})$ is a grid function which we assume to be decomposed into linear combinations with scalar factors $\varphi^{(g)}$ which carries the dependence on $\mathbf{u}$ and a skeleton or shape function $\hat{\boldsymbol{\Phi}}^{(g)}$, thus we write

$$
\boldsymbol{\Phi}_{K}(\tilde{\mathbf{u}})=\sum_{g} \varphi_{K}^{(g)}(\tilde{\mathbf{u}}) \hat{\boldsymbol{\Phi}}_{K}^{(g)}
$$

where the sum runs over a finite number of shape functions. The shape functions will be responsible for constraint preservation.

If we define a generic discrete constraint $\left.\widetilde{C}_{K} \cdot \mathbf{u} \approx \mathcal{C}(\mathbf{u})\right|_{K}$ where $\mathcal{C}$ is the analytic expression for the constraint, we can state the following lemma about constraint preservation.

LEMMA 3.1 (Constraint preservation). If the condition

$$
\widetilde{C}_{K} \cdot \hat{\boldsymbol{\Phi}}_{\widehat{K}}^{(g)}=0 \quad \forall K, \widehat{K}
$$

holds for the shape functions $\mathbf{\Phi}_{K}^{(g)}, g=1,2, \ldots$ of the flux distribution, then the resulting scheme (3.1) is locally constraint preserving.

The condition (3.3) represents the discrete analog of the respective vectordifferential identity. If the shape function $\boldsymbol{\Phi}_{K}^{(g)}$ is assumed to be unknown, the evaluation of (3.3) on several grid cells $K$ gives a homogeneous linear system whose nullspace provides constraint-preserving flux distributions. For details of the procedure see [18]. 


\section{2 curl-constraints on $2 d$ cartesian grids.}

We continue to present the results of the condition (3.3) in the case of a curlconstraint. We restrict ourselves to the two-dimensional Cartesian case and consider only the $z$-component of the curl when defining curl $\mathbf{u}=\partial_{x} u^{(y)}-\partial_{y} u^{(x)}$. The resulting flux distribution depends strongly on the choice of the constraint discretization. Similar to the case of a div-constraint the choice of central differences turned out to be not very fruitful, [18]. Instead we choose the extended operator

$$
\left.\widetilde{\operatorname{curl}}^{(\star)} \mathbf{u}\right|_{i, j}=\frac{\left\{u_{i+1, j}^{(y)}\right\}_{y}-\left\{u_{i-1, j}^{(y)}\right\}_{y}}{2 \Delta x}+\frac{\left\{u_{i, j-1}^{(x)}\right\}_{x}-\left\{u_{i, j+1}^{(x)}\right\}_{x}}{2 \Delta y}
$$

whose averaging

$$
\begin{aligned}
& \left\{\psi_{i, j}\right\}_{y}=\frac{1}{4}\left(\psi_{i, j+1}+2 \psi_{i, j}+\psi_{i, j-1}\right) \\
& \left\{\psi_{i, j}\right\}_{x}=\frac{1}{4}\left(\psi_{i+1, j}+2 \psi_{i, j}+\psi_{i-1, j}\right)
\end{aligned}
$$

introduce a multi-dimensional appearance. Neglecting the details we just present the results of the evaluation of the condition (3.3). Up to a factor the first element of the resulting nullspace represents a grid function $\hat{\mathbf{\Phi}}_{i, j}^{(1)}$ with mostly vanishing entries in the grid except for

$$
\begin{array}{ll}
\left.\hat{\mathbf{\Phi}}_{i, j}^{(1)}\right|_{i+1, j+1}=(\Delta y, \Delta x)^{T}, & \left.\hat{\boldsymbol{\Phi}}_{i, j}^{(1)}\right|_{i, j+1}=(-\Delta y, \Delta x)^{T}, \\
\left.\hat{\boldsymbol{\Phi}}_{i, j}^{(1)}\right|_{i, j}=(-\Delta y,-\Delta x)^{T}, & \left.\hat{\boldsymbol{\Phi}}_{i, j}^{(1)}\right|_{i+1, j}=(\Delta y,-\Delta x)^{T} .
\end{array}
$$

The nullspace is 4-dimensional and the elements $\hat{\mathbf{\Phi}}_{i, j}^{(2,3,4)}$ result from shifting of (3.6) around the center cell $(i, j)$. The upper right two sketches of Figure 3.1 show the structure of the flux distributions $\hat{\boldsymbol{\Phi}}_{i, j}^{(1)}$ and $\hat{\boldsymbol{\Phi}}_{i, j}^{(2)}$. Any scheme that is built from these flux distributions assembled as in (3.2) and plugged into (3.1) will exactly preserve the discrete curl-operator (3.4) during the entire calculation.

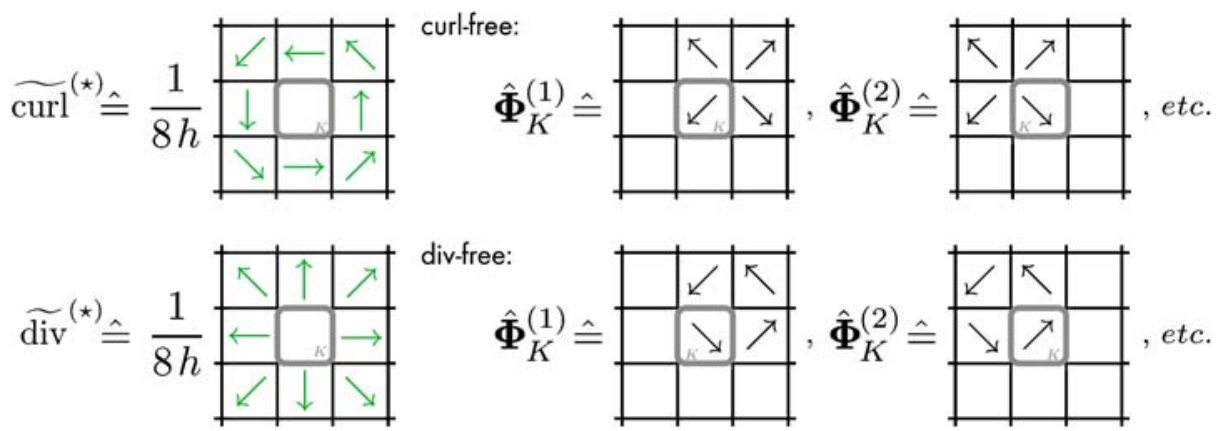

Figure 3.1: Discrete constraints coefficients and corresponding flux distributions for curl- and div-preservation, upper and lower row, respectively. 


\subsection{Dual flux distributions.}

The discrete curl-constraint (3.4) can be written in the general form

$$
\left.\widetilde{\operatorname{curl}} \mathbf{u}\right|_{i, j}=\sum_{-1 \leq k, l \leq 1} \mathbf{c}_{k, l} \cdot \mathbf{u}_{i+k, j+l}
$$

with appropriate vector-valued coefficients $\mathbf{c}_{k, l}$ in a $3 \times 3$ block around cell $(i, j)$. These coefficients are shown in the upper left corner of Figure 3.1. These vectors shall not be confused with the values of the flux distribution. Indeed, since the operator $\widetilde{\operatorname{curl}}_{i, j}$ is a functional on the space $V$ of vector valued grid functions the set $\mathbf{c}_{k, l}$ forms a representative of an element of the dual space $V^{\star}$. It is interesting to mention the result for the div-preservation in 2 dimensions in this context. The respective discrete operator and its flux distributions are depicted in the lower row of Figure 3.1. To some extent the objects have changed their role between the actual space and its dual. This relation may also be found on the analytic level.

\section{Curl-free methods for the wave equation system.}

Equipped with the curl-preserving flux distributions we are able to formulate a vorticity-preserving upwind finite volume scheme. We follow the ideas of [17].

\subsection{Flux formulation.}

Crucial for the vorticity preservation is the evolution of the momentum in (2.2). We assume a classical finite volume scheme based on intercell fluxes is given for (2.2), see [7]. Due to the gradient structure the update for the momentum has the form

$$
\mathbf{m}_{i, j}^{n+1}=\mathbf{m}_{i, j}^{n}+\frac{\Delta t}{\Delta x}\left(p_{i-\frac{1}{2}, j}-p_{i+\frac{1}{2}, j}\right)\left(\begin{array}{l}
1 \\
0
\end{array}\right)+\frac{\Delta t}{\Delta y}\left(p_{i, j-\frac{1}{2}}-p_{i, j+\frac{1}{2}}\right)\left(\begin{array}{c}
0 \\
1
\end{array}\right),
$$

where $p_{i+\frac{1}{2}, j}$ and $p_{i, j+\frac{1}{2}}$ are momentum components of the intercell flux, i.e., the pressure. They may be obtained using any approximate or exact Riemann solver for the wave equation system. With the definitions of the flux distributions

$$
\left.\mathbf{\Phi}_{i, j+\frac{1}{2}}^{\text {(class) }}\right|_{i, j}=\left.\frac{\Delta t}{\Delta y} p_{i, j+\frac{1}{2}}\left(\begin{array}{c}
0 \\
-1
\end{array}\right) \quad \mathbf{\Phi}_{i, j+\frac{1}{2}}^{\text {(clas) }}\right|_{i, j+1}=\frac{\Delta t}{\Delta y} p_{i, j+\frac{1}{2}}\left(\begin{array}{l}
0 \\
1
\end{array}\right)
$$

and analogous expressions for $\boldsymbol{\Phi}_{i+\frac{1}{2}, j}^{\text {(clas) }}$ the classical scheme (4.1) can be equivalently written in the form (3.1). However, the flux distributions in (4.2) are not curl-preserving since they are no linear combinations of (3.6).

We modify the update in the following way in order to obtain an exactly curlpreserving numerical method. Instead of using (4.2) we utilize the curl-preserving 
flux distributions (3.6) for a full flux distribution in the form

$$
\boldsymbol{\Phi}_{i, j+\frac{1}{2}}^{(\text {curl-free })}=\frac{\Delta t}{8 \Delta x \Delta y} p_{i, j+\frac{1}{2}}\left(\boldsymbol{\Phi}_{i, j}^{(1)}+\boldsymbol{\Phi}_{i, j}^{(2)}\right) .
$$

The distribution $\boldsymbol{\Phi}_{i+\frac{1}{2}, j}^{\text {(curl-free) }}$ is analogously formed from $\boldsymbol{\Phi}_{i, j}^{(1)}, \boldsymbol{\Phi}_{i, j}^{(4)}$ and $p_{i+\frac{1}{2}, j}$. The new flux distribution $\boldsymbol{\Phi}_{i, j+\frac{1}{2}}^{\text {(curlfree) }}$ is shown in Figure 4.1 together with its classical counterpart. Both distributions shown correspond to the update in $y$-direction. In the program the update denoted by "+ =" according to this distribution reads

$$
\begin{aligned}
\mathbf{m}_{i, j}^{n+1}+ & =\frac{\Delta t}{4 \Delta x} p_{i, j+\frac{1}{2}}(0,-1)^{T}, & \mathbf{m}_{i, j+1}^{n+1}+ & =\frac{\Delta t}{4 \Delta x} p_{i, j+\frac{1}{2}}(0,1)^{T} \\
\mathbf{m}_{i+1, j}^{n+1}+ & =\frac{\Delta t}{8 \Delta x} p_{i, j+\frac{1}{2}}(1,-1)^{T}, & \mathbf{m}_{i+1, j+1}^{n+1}+ & =\frac{\Delta t}{8 \Delta x} p_{i, j+\frac{1}{2}}(1,1)^{T} \\
\mathbf{m}_{i-1, j}^{n+1}+ & =\frac{\Delta t}{8 \Delta x} p_{i, j+\frac{1}{2}}(-1,-1)^{T}, & \mathbf{m}_{i-1, j+1}^{n+1}+ & =\frac{\Delta t}{8 \Delta x} p_{i, j+\frac{1}{2}}(-1,1)^{T}
\end{aligned}
$$

for a single $y$-flux calculated at the edge $\left(i, j+\frac{1}{2}\right)$. The analogous update is performed for all other edges of a cell. We implemented the scheme based on a Roe-solver as approximate Riemann solver. The input values for the numerical flux are obtained from a linear reconstruction using van-Leers limiter. The time integration followed a second order improved Euler method and a dimensional splitting.
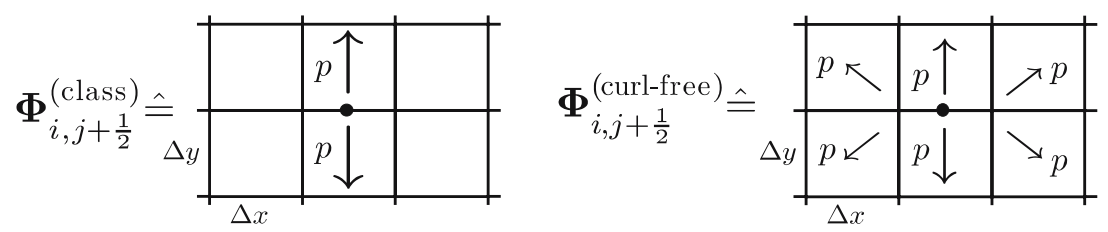

Figure 4.1: Flux distributions for the update of the momentum in the wave equation system. Left: a classical finite volume scheme. Right: vorticity-preserving flux distribution.

\subsection{D Riemann problem.}

The abilities of the new vorticity preserving scheme are demonstrated with the simulation of a $2 \mathrm{~d}$ Riemann problem. We write $\mathbf{m}=(m, n)$ and use $f(\rho)=\rho^{2}$ in the equations. The initial conditions are given by

$$
\left.(\rho, m, n)^{T}\right|_{t=0}=\left\{\begin{array}{lc}
(1,0,0)^{T} & x \geq 0 \text { and } y \geq 0 \\
\left(\rho_{\max }, m_{\max }, n_{\max }\right) & \text { else }
\end{array}\right.
$$

with $\rho_{\max }>1$ and

$$
m_{\max }=n_{\max }=\left(\rho_{\max }-1\right) \sqrt{\left(1+\rho_{\max }\right) / 2} .
$$


This Riemann problem is considered in [11] in the context of existence of solutions, see also [2]. Here, the computational domain is chosen to be $[-1,1]^{2}$, the end time $t=0.12, C F L=0.9$ and the initial maximal density $\rho_{\max }=9$.

In the initial conditions the momentum vector drops to zero along the positive coordinate axes producing a singularity in the vorticity distribution. Analytically, this singularity is perfectly preserved since curl $\mathbf{m}=$ const. However, the density jump together with a vorticity-free momentum discontinuity propagates across the computational domain. See Figure 4.2 for a plot of the initial conditions and a sketch of the solution at $t=0.12$.
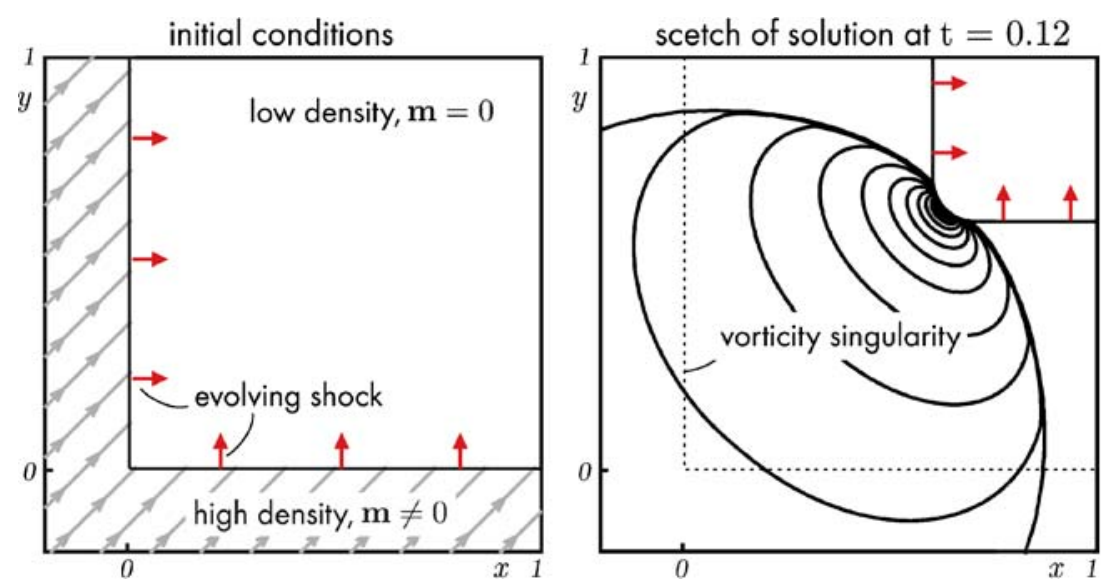

Figure 4.2: Left: initial conditions for the Riemann problem (4.5). Right: sketch of the density contours of the solution at $t=0.12$. The dotted lines indicate the position of the vorticity singularity.

Figure 4.3 shows the numerical result for the new curl-preserving fluxes (left) in comparison with the classical fluxes (right). The figure shows the contours of the magnitude of the momentum $\sqrt{m^{2}+n^{2}}$. The grid used $200 \times 200$ grid cells on $[-1,1]^{2}$ but the plot shows only the section $[-0.2,1]^{2}$ covered by $120 \times 120$ cells.

Both calculations are clearly different. The non-curl-preserving fluxes produce spurious oscillations along the diagonal path of the corner discontinuity. Furthermore, at the origin the classical method shows an unphysical nonsymmetric behavior which is cured by the use of the curl-preserving method. The deviations of both results are reflected in the vorticity distribution which is perturbed in the classical case but exactly preserving when using the new method. Figure 4.4 shows the contours of curl $\mathbf{m}$ obtained with the extended operator (3.4) for the solution of the classical and curl-free method. The classical scheme shows vorticity contamination along the path of the shock wave corner with values around $\approx \pm 100$. In the curl-free case only the vorticity singularities with values $\approx \pm 800$ are present. These are exactly preserved from the initial conditions, but influence the computational domain in the classical case. 

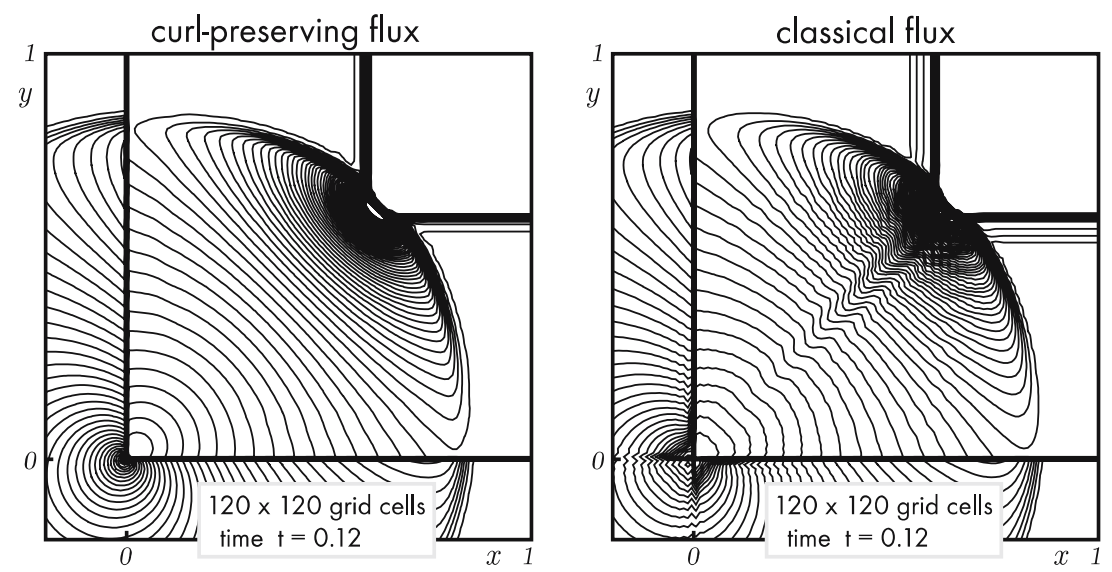

Figure 4.3: Contour plots for the magnitude of the momentum $\sqrt{m^{2}+n^{2}}$ in the solution of the Riemann problem (4.5) at time $t=0.12$. Left: Calculation with the curl-preserving method. Right: Result for a classical method.
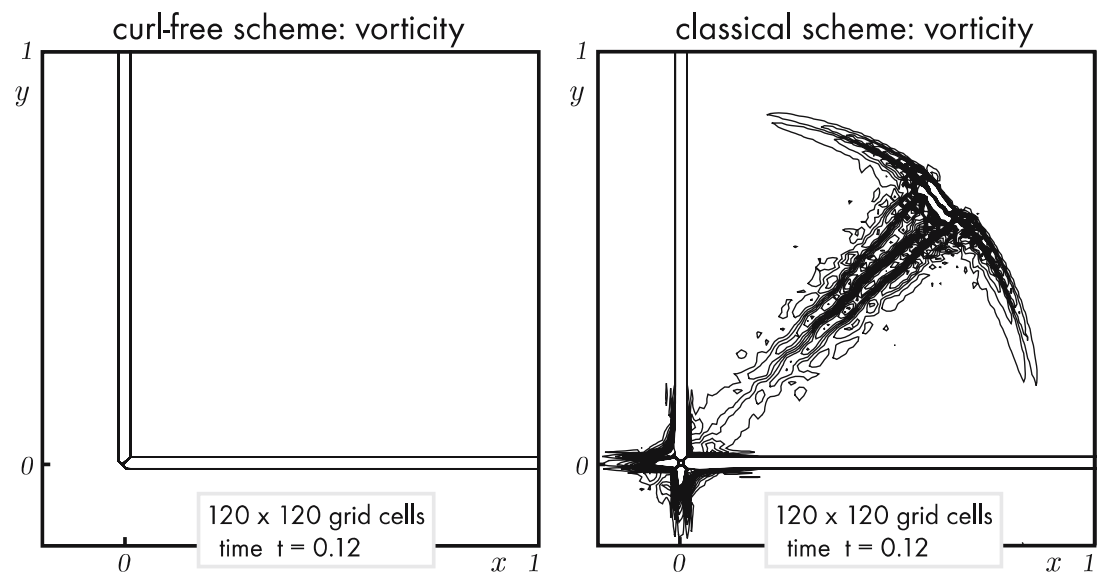

Figure 4.4: Contour plots for the value of the curl of momentum curl $\mathbf{m}$ in the solution of the Riemann problem (4.5) at time $t=0.12$. Left: Calculation with the curl-preserving method. Only the initial vorticity singularities are present. Right: Result for a classical method. The domain is spoiled by wrongly generated curl $\mathbf{m}$.

\section{Curl-free discretization for shallow water equations.}

The system of shallow water equations

$$
\begin{array}{r}
\partial_{t} \rho+\operatorname{div}(\rho \mathbf{v})=0 \\
\partial_{t}(\rho \mathbf{v})+\operatorname{div}\left(\rho \mathbf{v} \mathbf{v}^{T}\right)+\operatorname{grad} p=0
\end{array}
$$

with $p(\rho)=\frac{1}{2} g \rho^{2}$ has exactly the same form as the wave equations system up to the convective term in the second equation. However, this convective term makes 
the constraint preservation, i.e., the vorticity evolution, very hard to control. The main difficulty is the non-linear operation when deriving the vorticity equation, namely the transformation to Lagrangian form and division by density before taking the curl. A numerical discretization of the system (5.1) which reproduces a precise numerical counterpart of the vorticity evolution is work in progress. However, the above results for the wave equation system suggest that a proper discretization of the pressure gradient will have an influence on the behavior of the vorticity evolution.

Some numerical method use the velocity equation (2.8) to incorporate vorticity preservation, see [14]. Here, however, we concentrate on the conservative formulation (5.1) in order to handle shock waves correctly.

\subsection{Discretization.}

We proceed by extending the curl-free numerical method of the wave equation system to the shallow water equations. The equations have the form

$$
\partial_{t} U+\partial_{x} F(U)+\partial_{y} G(U)=0
$$

with the variable vector $U=\left(\rho, \rho v^{(x)}, \rho v^{(y)}\right)^{T}$ and flux functions

$$
F(U)=\left(\begin{array}{l}
\rho v^{(x)} \\
\rho v^{(x)} v^{(x)} \\
\rho v^{(x)} v^{(y)}
\end{array}\right)+\left(\begin{array}{c}
0 \\
p \\
0
\end{array}\right), \quad G(U)=\left(\begin{array}{c}
\rho v^{(y)} \\
\rho v^{(y)} v^{(x)} \\
\rho v^{(y)} v^{(y)}
\end{array}\right)+\left(\begin{array}{l}
0 \\
0 \\
p
\end{array}\right)
$$

These functions have been written such as to emphasise the two distinct influences, convection and pressure. The aim is to separate these influences also in the numerical method in order to apply the special curl-free flux distribution to the pressure gradient alone. Note, that such a separation has also been proved useful in the CUSP scheme of $[9,10]$.

Usually, in a standard finite volume update

$$
U_{i, j}^{n+1}=U_{i, j}^{n}+\frac{\Delta t}{\Delta x}\left(\widetilde{F}_{i-\frac{1}{2}, j}-\widetilde{F}_{i+\frac{1}{2}, j}\right)+\frac{\Delta t}{\Delta x}\left(\widetilde{G}_{i-\frac{1}{2}, j}-\widetilde{G}_{i+\frac{1}{2}, j}\right)
$$

the numerical flux functions $\widetilde{F}_{i+\frac{1}{2}, j}$ and $\widetilde{G}_{i, j+\frac{1}{2}}$ are highly involved. To gain margin for the modification of the discretization we consider the simple numerical flux

$$
\widetilde{F}_{i+\frac{1}{2}, j}=\frac{1}{2}\left(F\left(U_{i+1, j}\right)+F\left(U_{i, j}\right)\right)-\frac{1}{2} c_{i+\frac{1}{2}, j}^{(\max )}\left(U_{i+1, j}-U_{i, j}\right)
$$

which is the local Lax-Friedrichs flux. The diffusive part is multiplied with the maximal modulus of the local propagation speed. Obviously, this numerical flux decomposes into a true flux part and a diffusion part, $\widetilde{F}_{i+\frac{1}{2}, j}=\widetilde{F}_{i+\frac{1}{2}, j}^{(F)}+\widetilde{F}_{i+\frac{1}{2}, j}^{(D)}$. Accordingly inside the flux part the pressure influence can be identified as

$$
\widetilde{F}_{i+\frac{1}{2}, j}^{(F)}=\left(\begin{array}{l}
\rho v^{(x)} \\
\rho v^{(x)} v^{(x)} \\
\rho v^{(x)} v^{(y)}
\end{array}\right)_{i+\frac{1}{2}, j}+p_{i+\frac{1}{2}, j}\left(\begin{array}{l}
0 \\
1 \\
0
\end{array}\right),
$$


where the values at $\left(i+\frac{1}{2}, j\right)$ are evaluated as arithmetic average of the adjacent values as in the flux (5.5).

After isolating the pressure expression in the numerical flux (5.5) on one cell interface according to (5.6) it is easy to treat this part separately. Indeed this pressure expression enters the update exactly as the pressure of the wave equation system in (4.1). Hence, the same successful curl-free flux distribution as in (4.4) can be applied.

An extension to other numerical flux functions is possible once the numerical flux is written in the form (5.5) with an arithmetic average of the flux and a diffusive central difference part. Especially for numerical fluxes based on Roe solvers this is always possible. The basic assumption behind this approach is that the diffusive part does not influence the vorticity generation too much. This needs to be investigated in the future.

The numerical method described above for the shallow water equations was implemented based on the local Lax-Friedrichs flux, linear reconstruction and a Heun time integration method. Both linear reconstruction and time integration do not interfere with the special pressure flux distribution. The two dimensional operator was realized by an additive splitting in which both dimensions are updated during one step.

\subsection{Numerical example.}

The generation and evolution of vorticity in the shallow water case is difficult to check numerically. First, any vorticity is not frozen locally but is advected, hence, any vorticity pattern change with time. Furthermore, due to numerical viscosity, vorticity is generated even in smooth regions for a perfectly curl-free discretization. This viscous vorticity generation is as unavoidable as the viscous smearing of the shock wave in a inviscid calculation. However, this vorticity is of order of the grid size and vanishes in the limit of fine grids.

The vorticity generation of a shock wave is much stronger than viscous generation of a smooth solution due to the strong gradients. In the following we consider a shock wave running over a dense cloud and investigate the evolution of the vorticity field.

The initial condition for the density are depicted in the left hand side plot of Figure 5.1. It consists of a shock wave

$$
\rho(x, y)=\left\{\begin{array}{ll}
1.0 & x>x_{s} \\
8.0 & x<x_{s}
\end{array}, \quad v^{(x)}(x, y)= \begin{cases}0.0 & x>x_{s} \\
42.0 & x<x_{s}\end{cases}\right.
$$

at $x_{s}=-0.7$ which propagates with Mach number $M=6$ in $x$-direction. The values are derived from the Rankine-Hugoniot conditions (2.14). The $y$-component of the velocity vanishes entirely, $v^{(y)} \equiv 0$. The domain in front of the shock wave is disturbed by a circular density cloud around the origin. We choose an exponential decay, that is, the density difference

$$
\Delta \rho(x, y)=2 \exp \left(-\frac{x^{2}+y^{2}}{\varepsilon}\right)
$$



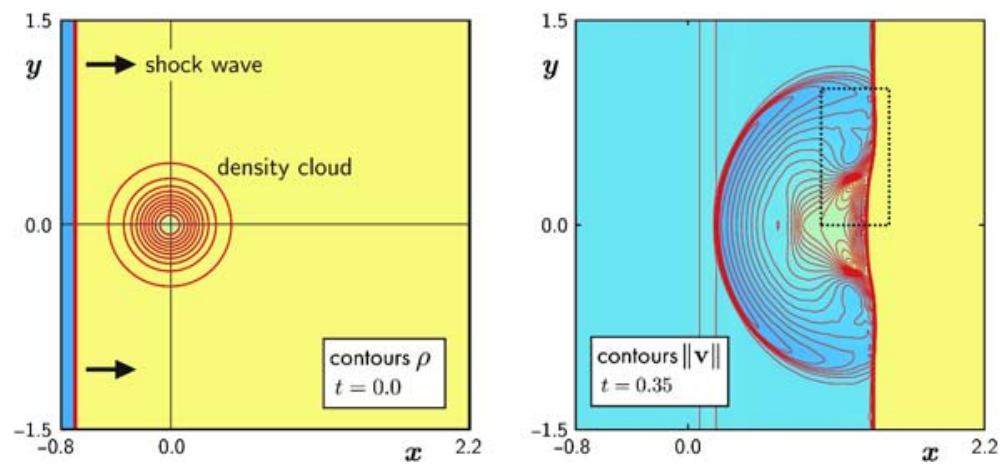

Figure 5.1: Shock wave interaction with a density cloud for the shallow water equations. Left: initial conditions for the density with shock wave and exponentially decaying density bump. Right: Contours of $\|\mathbf{v}\|$ at time $t=0.35$ after the shock passed through the cloud. The black box indicates the section shown in Figures 5.3 and 5.4.

with $\varepsilon=1 / 20$ is added to the initial conditions. When the shock hits the cloud a weaker shock wave is reflected and the shock slows down and bends back inside the cloud. The contours of the velocity magnitude $\|\mathbf{v}\|$ are shown in the right hand side plot of Figure 5.1 at time $t=0.35$. Two straight contours at the left edge stem from the initial conditions. The cloud itself is compressed and swept behind the shock wave.

The process is simulated in the domain $x \in[-0.8,2.2] \times[-1.5,1.5]$, up to a time $t=0.35$. We used the second order scheme based on the local LaxFriedrichs flux with both the classical and curl-free pressure discretization. The CFL number was $C F L=0.95$ in all cases. We show the results for the grid sizes $200 \times 200$ with $\Delta x=0.015$ and $400 \times 400$ with $\Delta x=0.0075$.

Due to the two-dimensional character of the simulation the vorticity is a scalar $\omega=\partial_{x} v^{(y)}-\partial_{y} v^{(x)}$. The time evolution of the maximal value and the integral of $|\omega|$ give some insight in the vorticity generation of the process. We calculate the values of $\|\boldsymbol{\omega}\|_{\infty}$ and $\|\boldsymbol{\omega}\|_{1}$ from the simulation results at $t=$ $0.05,0.1,0.15,0.2,0.25,0.3$, and 0.35 and plot their evolution as piecewise linear curve in Figure 5.2. The figure shows the results of the classical and the curl-free scheme both for grids with $N=200$ and $N=400$.

The maximal value of $|\omega|$ becomes stationary once the shock has passed through the cloud at $t \approx 0.2$. However, the value is $60 \%$ higher for the case $N=400$ due to higher resolution and less numerical diffusion. The curve for the integral of $|\omega|$ shows a non-monotone behavior. The difference between the different resolutions is less pronounced. Both schemes, classical and curl-free, exhibit approximately the same behavior for the overall generation of vorticity. The difference of the schemes becomes evident in the details of the vorticity structure as discussed below.

The expression for the vorticity generation across a shock wave (2.20) allows to derive a rough estimate how much vorticity is to expect in the present process following the presentation in [12]. The shock wave constantly generates vorticity 

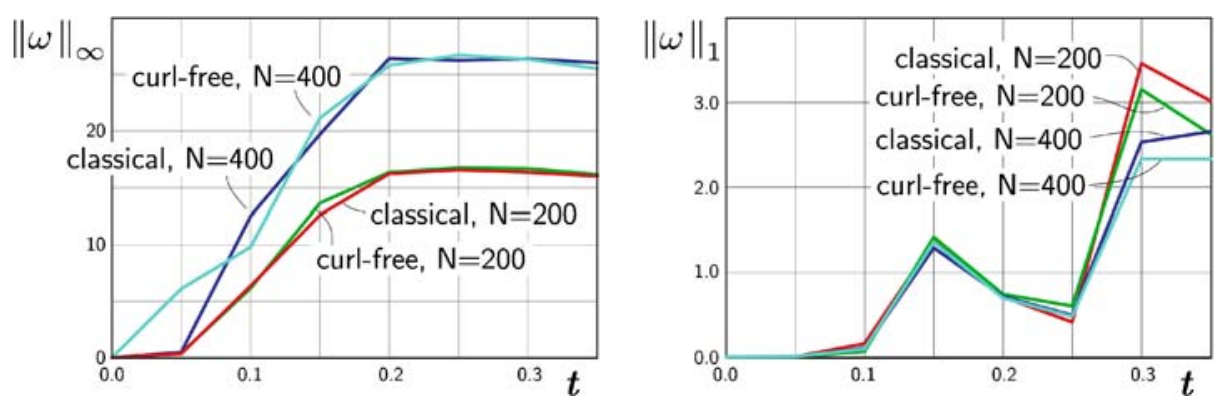

Figure 5.2: Time evolution of the maximal value (left) and the integral (right) of vorticity $|\omega|$ for the shock wave cloud interaction. The plots show the results for the classical and curl-free scheme at two different resolutions.

according to the value of $\partial_{y} \rho$ while it is running over the density cloud. If we assume that the velocity jump across the shock does not change during the passage the vorticity gain is simply given by

$$
\omega(x, y)=\frac{\partial_{y} \rho(x, y)}{\rho(x, y)}\left[v^{(x)}\right]
$$

where the density distribution is given by (5.8). This is based on the additional assumption that the vorticity does not change after it is created by the shock which is not true due to advection. However, the result enables us to give the rough estimates $\|\boldsymbol{\omega}\|_{\infty} \approx 19.8$ and $\|\boldsymbol{\omega}\|_{1} \approx 5.2$ for the current process. These values are in approximate correspondence with the results of the numerical simulations.

Let us consider the vorticity structure in more detail. The vorticity generated by the shock performs a complicated movement behind the shock due to compression and advection. The vorticity patterns are symmetric with respect to the $x$-axis. At time $t=0.35$ they are concentrated in the area $[1.0,1.5] \times[-1.0,1.0]$. Due to symmetry we restrict ourselves to $\Omega=[1.0,1.5] \times[0.0,1.0]$. In the right hand side plot of Figure 5.1 this area is indicated by a black box.

Figure 5.3 shows the contours of vorticity $\omega$ at time $t=0.35$ inside the section $\Omega$ based on the curl-free (left) and classical (right) numerical method. Both plots were obtained from a simulation with $N=200$. The shock wave cuts both plots on the left half bending towards the middle in the lower half. The strong vorticity generation along the shock line is visible. The overall patterns of the vorticity look the same for both calculations. However, the result differ in many details. The difference is more pronounced than in the results for density or velocity. This shows that the vorticity is highly sensible to the way of discretization inside the numerical method.

The curl-free method leads to more distinct features along the shock line. Figure 5.3 shows more clear vortex patterns, i.e., more isolated vorticity extrema. This phenomenon is even more visible in the high resolution result in Figure 5.4 which shows the same section $\Omega$ for both schemes at $t=0.35$ but based on a 

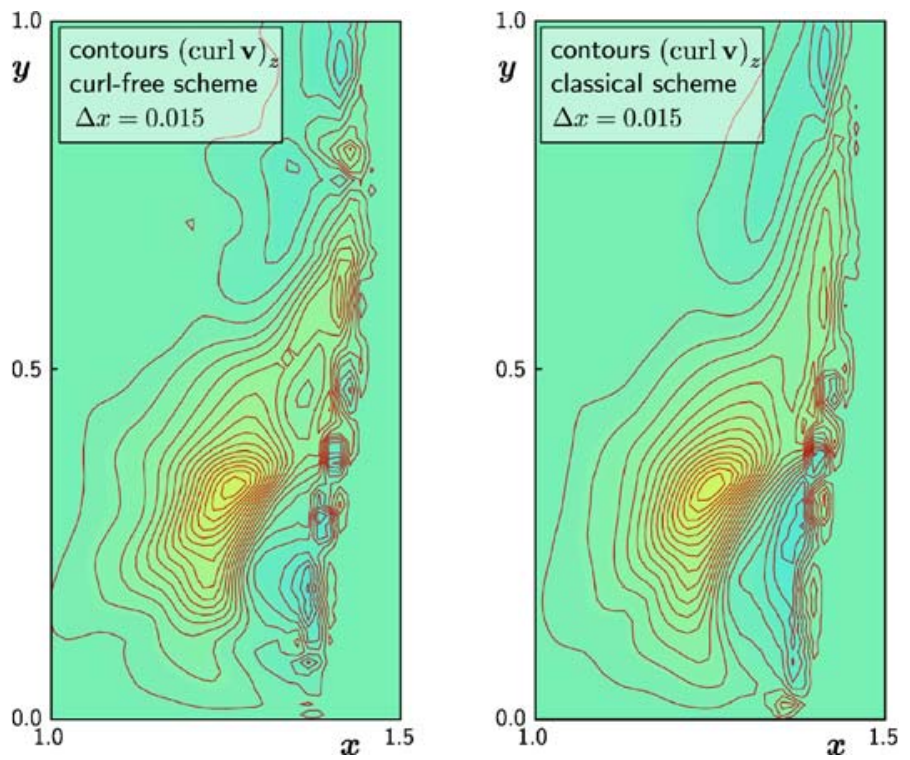

Figure 5.3: Vorticity contours in the section $\Omega=[1.0,1.5] \times[0.0,1.0]$ at time $t=0.35$ for the shock wave interaction with a dense cloud. Left: curl-free discretization. Right: classical discretization. Both results used $x=0.015$ which leads to a $33 \times 66$ grid for the section shown. Again, the curl-free result shows a more detailed structure.
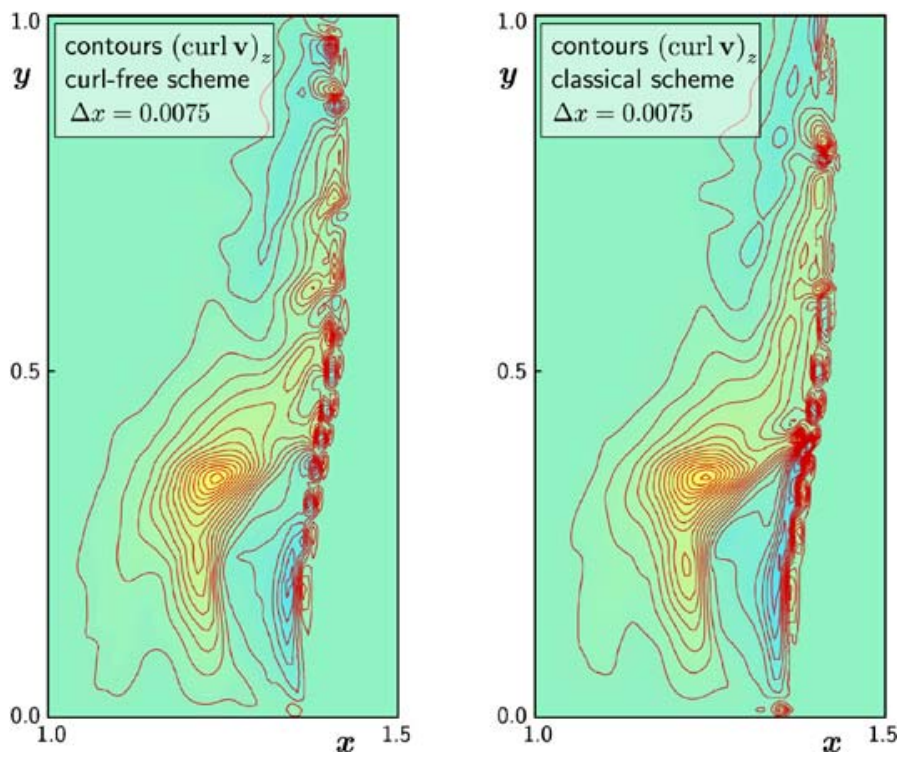

Figure 5.4: Vorticity contours in the section $\Omega=[1.0,1.5] \times[0.0,1.0]$ at time $t=0.35$ for the shock wave interaction with a dense cloud. Left: curl-free discretization. Right: classical discretization. Both results used $x=0.0075$ which leads to a $66 \times 133$ grid for the section shown. Again, the curl-free result shows a more detailed structure. 
$N=400$ calculation. Here the classical result shows a smeared vorticity along the lower part of the shock line, while the curl-free calculation gives distinct vertexes.

\section{Conclusion.}

The intrinsic constraints of the wave equation system and the system of inviscid shallow water equations have been discussed and specialized curl-free numerical method have been derived and investigated for these systems.

The pure gradient operator in the moment equation of the wave equation system leads to a curl-constraint in the sense that the curl of the moment variable does not change during any evolution. In the inviscid shallow water system the moment equation is supplemented by a convection term which spoils the clear curl-constraint. Instead, a generalized constraint based on vorticity can be derived. In smooth solutions vorticity can not be created and any vorticity is only advected. However, shock waves can create vorticity.

In the numerical method the pressure gradient flux of the equations require a special treatment based on the flux distribution method derived in [18]. In order to apply the method to the shallow water system, the pressure flux needs to be isolated by rearranging the numerical flux. The constraint-preserving flux distribution provides a conservative finite volume update which exactly preserves the value of a discrete constraint operator. The flux modification is easily implemented in any finite volume frame work and does not interfere with data reconstruction or time integration.

The curl-free discretization of the wave equation system leads to the reduction of spurious oscillations and an asymmetric solution. Due to the generalized character of the vorticity constraint in the shallow water system the influence of the curl-free discretization is more difficult to detect. Nevertheless, it could be shown that vortex pattern are more clearly resolved with the new curl-free discretization.

\section{REFERENCES}

1. J. U. Brackbill and D. C. Barnes, The effect of nonzero $\nabla \cdot B$ on the numerical solution of the magnetohydrodynamic equations, J. Comput. Phys., 35 (1980), p. 426-430.

2. S. Canic, B. L. Keyfitz, and E. H. Kim, A free boundary problem for a quasi-linear degenerate elliptic equation: Regular reflection of weak shocks, Commun. Pure Appl. Math., 55 (2002), pp. 71-92.

3. C. M. Dafermos, Quasilinear hyperbolic systems with involutions, Arch. Ration. Mech. Anal., 94 (1986), p. 373-399.

4. C. R. Evans and J. F. Hawley, Simulation of magnetohydrodynamic flows: a constrained transport method, Astrophys. J., 332 (1988), p. 659-677.

5. M. Fey and M. Torrilhon, A constrained transport upwind scheme for divergence-free advection, in Hyperbolic Problems: Theory, Numerics and Applications, Proc. 9th Intl. Conf. Hyperbolic Problems, USA 2002, Conference Proceedings, Springer, New York, 2003. 
6. M. Fey and M. Torrilhon, Vorticity preserving finite volume method for the wave equation system, in Hyperbolic Problems: Theory, Numerics and Applications, Proc. 10th Intl. Conf. Hyperbolic Problems, Japan 2004, Conference Proceedings, Yokohama Publishers, Yokohama, 2005.

7. E. Godlewski and P.-A. Raviart, Numerical Approximation of Hyperbolic Systems of Conservation Laws, Springer, New York, 1996.

8. J. M. Hyman and M. Shashkov, Natural discretizations for the divergence, gradient, and curl on logically rectangular grids, Comput. Math. Appl., 33 (1997), p. 81-104.

9. A. Jameson, Analysis and design of numerical schemes for gas dynamics, 1: artificial diffusion, upwind biasing, limiters and their effect on accuracy and multigrid convergence, Int. J. Comput. Fluid Dyn., 4 (1995), p. 171-218.

10. A. Jameson, Analysis and design of numerical schemes for gas dynamics, 2: artificial diffusion and discrete shock structure, Int. J. Comput. Fluid Dyn., 5 (1995), p. 1-38.

11. B. L. Keyfitz, Free Boundary Problems for Nonlinear Wave Equations: Interacting Shocks, Workshop on Multiphase Fluid Flows and Multi-Dimensional Hyperbolic Problems, Newton Institute, Cambridge, 2003.

12. P. Kornreich and J. Scalo, The galactic pump: a source of supersonic internal motions in the cool interstellar medium, Astrophys. J., 531 (2000), p. 366-383.

13. R. J. LeVeque, Finite Volume Methods for Hyperbolic Problems, Cambridge University Press, Cambridge, 2002.

14. S.-J. Lin and R. B. Rood, An explicit flux-form semi-Lagrangian shallow-water on the sphere, Q. J. R. Meteor. Soc. (Part B), 123 (1997), p. 2477-2498.

15. K. W. Morton and P. L. Roe, Vorticity-preserving Lax-Wendroff-type schemes for the system wave equation, SIAM J. Sci. Comput., 23 (2001), p. 170-192.

16. J. C. Nedelec, Mixed finite elements in $\mathbb{R}^{3}$, Numer. Math., 35 (1980), p. 315-341.

17. M. Torrilhon, Locally divergence-preserving upwind schemes for magnetohydrodynamic equations, SIAM J. Sci. Comput., 26 (2005), pp. 1166-1191.

18. M. Torrilhon and M. Fey, Constraint-preserving upwind methods for multidimensional advection equations, SIAM J. Numer. Anal., 42 (2004), p. 1694-1728.

19. G. Toth, The $\nabla \cdot B$ constraint in shock-capturing magnetohydrodynamics codes, J. Comput. Phys., 161 (2000), p. 605-652.

20. C. Truesdell, The Kinematics of Vorticity, Indiana University Press, Bloomington, 1954. 